\title{
Survival, functional outcome and satisfaction of first revision total knee arthroplasty at a mean eleven-year follow-up
}

\author{
Andreas Hecker ${ }^{1}$ (D) $\cdot$ Hans-Jürg A. Pütz $^{1} \cdot$ Sebastian Wangler ${ }^{1} \cdot$ Frank M. Klenke $^{1}$
}

Received: 29 October 2021 / Accepted: 5 January 2022 / Published online: 18 January 2022

(c) The Author(s) 2022

\begin{abstract}
Purpose Providing long-term outcome data after rTKA and compare one- versus two-stage and septic versus aseptic revisions.

Methods This study represents a single-center retrospective study of first rTKAs performed for any reason with a final follow-up of a minimum of five years. Outcome parameters included stability assessment ROM, radiologic assessment, HSS score, KSS score, OKS score, EQ-5D-3L and VAS. 44 patients were included in the study. Subgroups analysis of one- versus two-stage revision and septic versus aseptic revision was performed.

Results The leading causes of rTKA in this mean 11 year follow-up study were aseptic loosening (36\%) and periprosthetic joint infection (27\%). At the final follow-up, there was a $89 \%$ survivorship of the implants. Patients showed a ROM of $114 \pm 13^{\circ}$, HSS score of $78 \pm 12$, KKS objective score of $77 \pm 16$, KSS expectation and satisfaction score of $32 \pm 11$, KSS functional activity score of $50 \pm 20$, OKS of $30 \pm 9$, VAS of $53 \pm 25$ and EQ-5D index of 0.649 . Functional outcome scores were not significantly altered in the analyzed subgroups.

Conclusions In our 11 years follow-up, we obtained $89 \%$ implant survivorship. Measurements regarding functional outcome and pain showed results in the medium range of the respective scores, while patient satisfaction lay in the upper third. No significant differences in outcome scores between one- and two-stage revisions and septic versus aseptic revisions were observed. Level of Evidence Level III, retrospective cohort study.
\end{abstract}

Keywords Knee $\cdot$ Arthroplasty $\cdot$ Revision $\cdot$ Cause $\cdot$ Outcome $\cdot$ Satisfaction

\section{Introduction}

Total knee arthroplasty (TKA) represents a well-established approach for the treatment of advanced degenerative arthritis. Based on register data available in many countries, the reported survivorship of primary TKA is at $82 \%$ at 20 -year and $70 \%$ at 25-year follow-up [8]. Fostered by these good long-term results, indications for TKA have been expended in the past years. Especially the age at primary implantation has dropped, resulting in a more active patient population requesting a higher performance of the used implant. The number of TKA procedures is therefore expected to increase by $140 \%$ in 2050 [11]. Importantly, patients younger than

Andreas Hecker

andreas.hecker@insel.ch

1 Department of Orthopaedic Surgery and Traumatology, Inselspital, Bern University Hospital, University of Bern, Freiburgstrasse 4, 3010 Bern, Switzerland
65 years will represent the major recipients of primary TKA [18]. The aim of TKA is the long-term relief of pain and restoration of function. Unfortunately, knee replacements fail for various reasons, including aseptic loosening and infection, followed by instability, wear, and pain [6]. In this respect, younger age has been associated with a higher risk of aseptic mechanical failure after TKA. Considering the growing number of performed procedures and expended target population, orthopedic surgeons will be confronted with an increasing number of patients in need of revision TKA (rTKA). Some authors estimate that the number of rTKA will increase by up to $600 \%$ in 2030 [18].

While functionality and patient satisfaction following primary TKAs has been investigated thoroughly, data about the outcome after rTKA is limited. Primary TKA has been associated with a satisfaction rate of about $80 \%$. Some studies report similar outcomes following rTKA, while others report limited success $[13,27,30]$. An explanation for these heterogeneous results might be a different overall health 
status of the respective cohorts. Interestingly, the reason for revision surgery seems to influence the outcome. Revision TKA following aseptic loosening was associated with a better outcome than revision due to instability, malposition, or septic loosening [32].

Despite the indication, revision surgery is always challenging because many factors, including bone loss, ligamentous instability, and soft tissue problems, need to be considered. Late infection represents a challenging situation as it often requires a two-staged revision with primary infect control and secondary rTKA. The patients' immobilization between the two steps results in loss of muscle and is associated with a prolonged rehabilitation [24]. In general results after rTKA are thought to be worse than after primary TKA due to scaring, a tendency to develop patella baja, muscular imbalance and change of the biomechanics due to revision implants and/or bone loss.

This study aimed to analyze the outcome and implant survival of patients undergoing the first TKA revision, focusing on subjective satisfaction after a minimum follow-up of five years. We hypothesized that functional outcome following one- and two-stage revisions as well as after septic or aseptic revision does not differ after a minimum follow-up of five years.

\section{Material and methods}

This study represents a single-center retrospective study of rTKA performed at our institution between January 2000 and December 2012. The local ethics committee approved the study.

Inclusion criteria were first rTKA performed for any reason with a well-documented final follow-up of a minimum of five years and available outcome scores (Hospital of Special Surgery Score (HSS), Knee Society Score (KSS),
Oxford Knee Score (OKS), EQ-5D-3L, Visual Analog Scale (VAS)) [5, 7, 12]. Exclusion criteria were re-revisions (if first revision was not performed at our institution during the observational period), only exchange of the polyethylene inlay due to wear and secondary patellar resurfacing. Database evaluation revealed a total of 80 rTKAs eligible for this study. Thirty-six patients had to be excluded after application of the above mentioned exclusion criteria. Finally, 44 patients matched the inclusion criteria and were included in the study (Fig. 1).

The medical history, including the surgical report, was analyzed. The reason for revision, time to revision and the implant model used for revision were documented. Age, American Society of Anesthesiologists (ASA) score, height, and weight were raised. Clinical evaluation was performed focusing on stability and range-of-motion (ROM). Radiographic evaluation, including long-leg, standing anteroposterior and lateral views, was performed to detect implant loosening signs and evaluate the leg axis. Loosening was defined as a gap larger than $2 \mathrm{~mm}$ in the bone-cement- or bone-implant-interface [17]. In the OKS, the best value is 12 and the worst 60 . All other scores range from 0 to their maximum, with the latter representing the best reachable score.

A subgroup of the cohort $(N=16)$ was not available for physical and radiological examination at the final follow-up, but outcome scores could be raised and surgical history was present; therefore, those patients were also included for the respective analysis.

\section{Statistical analysis}

Statistical analyses were conducted in SPSS (IBM SPSS Statistics, Version 25 for Windows) with $P<0.05$ considered statistically different. Data normality was checked with the Kolmogorov-Smirnov test. When the normality assumption was satisfied, a student $t$-test was chosen to compare

Fig. 1 Patient selection chart

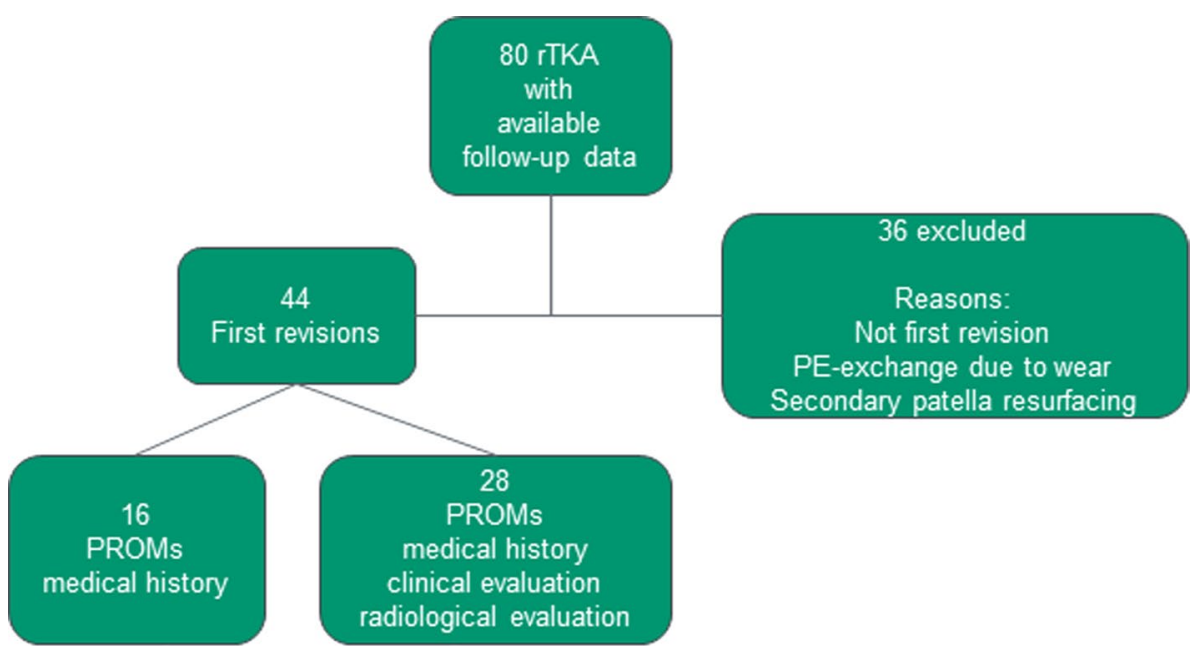


different subgroups. Data are given as mean and standard deviation. Kaplan-Meier survival was calculated with endpoints re-revision and implant survival.

\section{Results}

The present retrospective single-center study reports the outcome of 44 patients following first rTKA ( $50 \%$ female, average age $63 \pm 8$ years at rTKA). The right knee was affected predominantly in $61 \%$ of the cases. Regarding the overall population health status, 33 cases were classified ASA 2 and 11 ASA 3 . The body mass index (BMI) was $29 \pm 5 \mathrm{~kg} /$ $\mathrm{m}^{2}$. The mean follow-up was $11 \pm 3$ years. The mean time between first TKA and revision surgery (first revision) was $5 \pm 6$ years. All implants used for the primary TKA were standard cruciate retaining or posterior stabilized implants. The reasons for revision were loosening (36\%), infection (27\%), persistent pain (20\%), instability (10\%), and component malrotation (7\%). In 24 cases, a one-stage exchange was performed, while in 20 cases, a two-stage exchange was chosen. In eight of the two-stage exchanges, a preoperatively suspected infection could not be confirmed by intraoperative tissue samples and sonication. In 25 cases a condylar constrained implant was used, in 12 cases a hinge design was chosen and in seven cases a standard implant was utilized (Fig. 2, Table 1).

In six cases (14\%), a re-revision was necessary after a mean of $5 \pm 5$ years. Indications for re-revision with complete implant exchange were infection, trauma and pain without obvious reason in one case each. The implant was retained in three cases of secondary patellar resurfacing. Of the six re-revision cases, two secondary patella resurfacing cases needed a third revision with implant removal due to instability 7 months and loosening 32 months after rerevision. The cumulative Kaplan-Meier implant survival estimations for the endpoint re-revision is displayed in Fig. 3 and for the endpoint implant removal in Fig. 4. The implant survival at a mean of 11 years was $89 \%$ in this study.

In 28 cases, complete follow-up with the clinical and radiological examination was available, and in 16 cases, only outcome scores and medical history could be obtained.

\section{Radiological outcome}

Twenty-six patients had a mean anatomical valgus of $5 \pm 3^{\circ}$, and two patients had a mean varus axis of $4 \pm 0^{\circ}$. In 2 cases, a radiolucent line of more than $2 \mathrm{~mm}$ was detected on the radiographs, and therefore loosening was diagnosed.

\section{Functional outcome}

At the final follow-up, 8 patients showed limited medio-lateral instability of less than $5 \mathrm{~mm}$. In 20 patients, no mediolateral instability was detected. In 3 patients, an anteroposterior instability of less than $5 \mathrm{~mm}$ was found, while 25 cases were stable. The mean range of motion was $114 \pm 13^{\circ}$.

The mean HSS score was $78 \pm 12$. The mean HSS symptoms score was $39 \pm 10$, the mean HSS objective score was $41 \pm 5$. The mean KSS overall score was $77 \pm 16$, subdivided in an objective score of $62 \pm 15$ and a symptoms score (including patients with no objective measurements) of $16 \pm 6$. The mean KSS expectation and satisfaction score was $32 \pm 11$. The mean KSS functional activity score was $50 \pm 20$. The mean OKS score was $30 \pm 9$. The mean EQ-5D VAS level was $53 \pm 25$. The mean EQ-5D index was $0.649 \pm 0.173$.

\section{Subgroup analysis}

Subgroups were analyzed to test for differences regarding one or two-stage revision strategy (Table 2) and septic versus aseptic revisions (Table 3). No significant differences

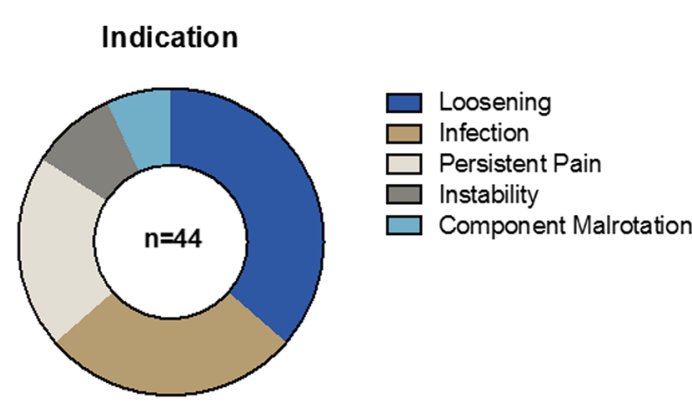

Fig. 2 Indications for revision TKA and implant type at the final follow-up. In the left pie-chart, the indications that lead to the first revision TKA are displayed. The biggest group was loosening (blue) followed by infection (brown). In the right pie-chart the implant type

\section{Prothesis Type}

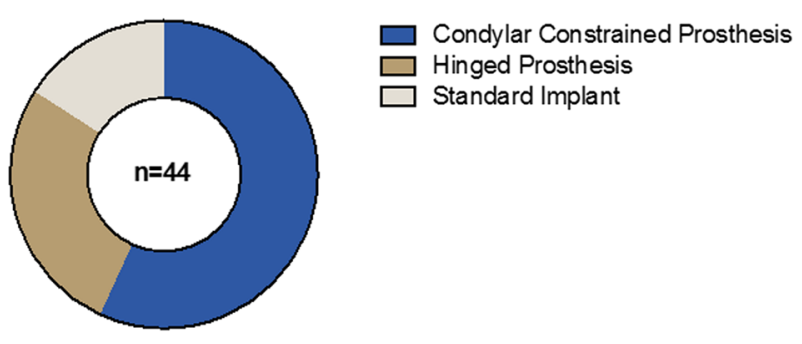

at the final follow-up is shown. The biggest group consists of condylar constrained implants (blue) followed by hinged implants (brown) (color figure online) 
Table 1 Demographic data, radiological and functional outcome of all included patients

Nender
Age at index surgery (years)
Age at rTKA (years)
Age at follow-up (years)
ASA classification

ASA classification

BMI $\left(\mathrm{kg} / \mathrm{m}^{2}\right)$

Affected side

Time to revision after index TKA (years)

Cause of rTKA

Approach

Re-rTKA

Re-Re-rTKA

Mean follow-up (years)

Implant at follow-up

Radiological outcome at follow-up

Varus axis $\left({ }^{\circ}\right)$

Valgus axis $\left({ }^{\circ}\right)$

Loosening (radiolucent line $>2 \mathrm{~mm}$ )

Functional outcome at follow-up

$\mathrm{ROM}\left({ }^{\circ}\right)$

Medio-lateral stability

Antero-posterior stability

HSS score

KSS score

KSS expectation and satisfaction score

KSS functional activity score
44

Female

$50 \%(22 / 44)$

Male

$50 \%(22 / 44)$

$58 \pm 9(44)$

$63 \pm 8(44)$

$74 \pm 9(44)$

ASA 1

$0 \%(0 / 44)$

ASA 2

$75 \%(33 / 44)$

ASA 3

$25 \%(11 / 44)$

ASA 4

$0 \%(0 / 44)$

$29 \pm 5(44)$

Right

$61 \%(27 / 44)$

Left

$39 \%(17 / 44)$

$5 \pm 6$ years $(44)$

Loosening

$36 \%(16 / 44)$

Infection

$27 \%(12 / 44)$

Persistent pain

$20 \%(9 / 44)$

Instability

$10 \%(4 / 44)$

Component malrotation

$7 \%(3 / 44)$

One-stage

$55 \%(24 / 44)$

$45 \%(20 / 44)$

$14 \%(6 / 44)$

$33 \%(2 / 6)$

$11 \pm 3(44)$

Condylar constrained prosthesis

$57 \%(25 / 44)$

Hinged prosthesis

$27 \%(12 / 44)$

$16 \%(7 / 44)$

28

$4 \pm 0(2)$

$5 \pm 3(26)$

$7 \%(2 / 28)$

28

$114 \pm 13(28)$

No instability

Instability $<5 \mathrm{~mm}$

Instability $>5 \mathrm{~mm}$

$71 \%(20 / 28)$

$29 \%(8 / 28)$

$0 \%(0 / 28)$

No Instability

Instability $<5 \mathrm{~mm}$

Instability $>5 \mathrm{~mm}$

$89 \%(25 / 28)$

$11 \%(3 / 28)$

$0 \%(0 / 28)$

$78 \pm 12(28)$

HSS objective

HSS symptoms

$41 \pm 5$ (28)

$39 \pm 10$ (44)

$77 \pm 16$ (28)

$62 \pm 15$ (28)

$16 \pm 6$ (44)

$32 \pm 11$ (44)

$24 \pm 9$ (44)

$9 \pm 3$ (44)

$50 \pm 20$ (44)

$19 \pm 9(44)$ 
Table 1 (continued)

\begin{tabular}{lll}
\hline & KSS standard activities & $17 \pm 7(44)$ \\
& KSS advanced activities & $7 \pm 6(44)$ \\
& KSS discretionary activities & $7 \pm 5(44)$ \\
OKS (12= best result, 60 = worst result) & & $30 \pm 9(44)$ \\
EQ-5D VAS & & $53 \pm 25(44)$ \\
EQ-5D Index (Reference: German value set) & & $0.649 \pm 0.173(44)$ \\
\hline
\end{tabular}

$\mathrm{ASA}=$ American society of anesthesiologists; $\mathrm{BMI}=$ Body mass index; TKA = Total knee arthroplasty; rTKA = Revision total knee arthroplasty; ROM=Range of motion; HSS = Hospital for special surgery score; $\mathrm{KSS}=$ Knee society score; $\mathrm{OKS}=$ Oxford knee score; $\mathrm{EQ}-5 \mathrm{D}=$ Measure of health-related quality of life

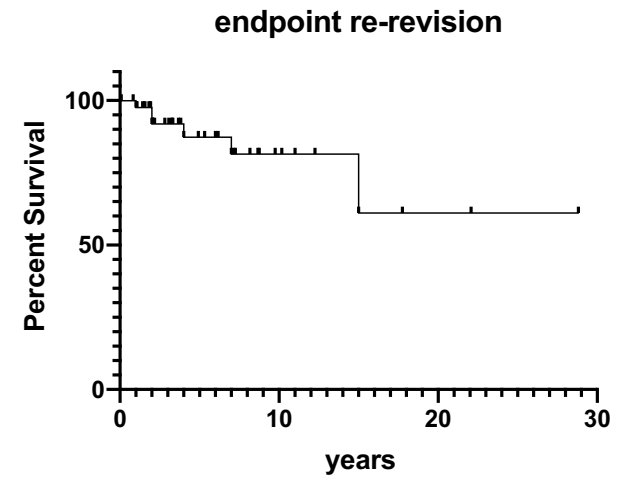

Fig. 3 Kaplan-Meier survival with the endpoint re-revision. The $x$-axis gives the follow-up in years and the $y$-axis the percent survival

were found regarding functional outcome scores between one- and two-stage approaches. [Table 2; HSS symptoms score $(p=0.748)$, KSS symptoms score $(p=0.338)$, KSS expectation and satisfactions score $(p=0.346)$, KSS functional score $(p=0.524)$, OKS score $(p=0.137)$ and EQ-5D VAS level $(p=0.474)]$.

The comparison of aseptic and septic revisions did not reveal significant differences regarding outcome scores as well. [Table 3; HSS symptoms score ( $p=0.625)$, KSS symptoms score $(p=0.624)$, KSS expectation and satisfactions score $(p=0.241)$, KSS functional score $(p=0.275)$, OKS score $(p=0.183)$ and EQ-5D VAS level $(p=0.515)]$.

\section{Discussion}

This study reports the indications, used implants, and outcomes of first revision TKAs in 44 patients treated between January 2000 and December 2012 at a university hospital. The mean follow-up time was 11 years, and the mean time between first TKA and revision TKA was five years. This is slightly lower than a mean of seven years to the first revision reported by previous studies [26, 31]. We could confirm our hypothesis that there is no differences in long-term outcome of one- and two-stage revision strategies as well

\section{endpoint implant removal}

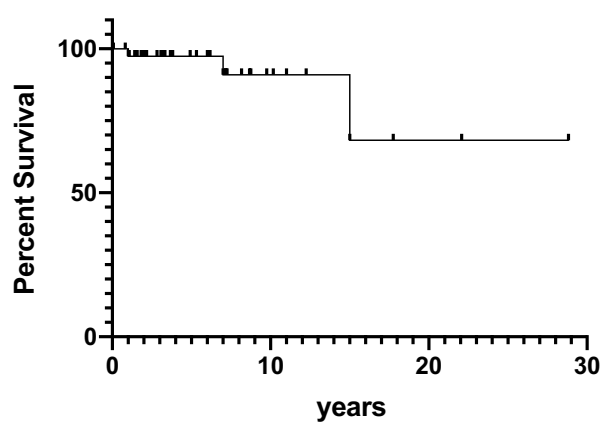

Fig. 4 Kaplan-Meier survival with the endpoint implant removal. The $x$-axis gives the follow-up in years and the $y$-axis the percent survival

as between septic and aseptic revisions. However, the latter results should be interpreted with caution with respect to the limited number of patients in the analyzed subgroups.

In the here presented population, the leading cause for revision surgery was aseptic loosening (36\%) and periprosthetic joint infection (27\%). This is in line with the findings by other published reports where both aseptic loosening and infection were listed among the first indications for rTKA $[26,28,29]$. The here presented cases show a high prevalence of infections and two-stage revisions which shows that rather complex cases where treated in our university center, while standard cases where rather treated in rural hospitals.

Complex cases with patients with higher comorbidities are associated with a higher risk of complications, prolonged hospitalization, and higher mortality $[3,16]$. Treatment often requires a two-staged procedure, and the support of an interdisciplinary team usually only available in big centers [2].

The outcome scores and clinical results of this study are within the known range. Successful treatment thresholds of the KSS range between 72 and 86 one year after primary TKA [14]. After revision TKA, the KSS has been reported to range from 70 to 77 points $[9,22]$. Our value of 77 points confirms these findings. The HSS score represents the satisfaction after TKA and has been reported 
Table 2 Demographic data, radiological and functional outcome one-stage vs. two-stage

\begin{tabular}{|c|c|c|c|c|}
\hline & & One-stage & Two-stage & $p$ \\
\hline $\mathrm{N}$ & & 24 & 20 & \\
\hline \multirow[t]{2}{*}{ Gender } & Female & $50 \%(12 / 24)$ & $50 \%(10 / 20)$ & \\
\hline & Male & $50 \%(12 / 24)$ & $50 \%(10 / 20)$ & \\
\hline Age at index surgery (years) & & $57 \pm 10(24)$ & $58 \pm 7(20)$ & \\
\hline Age at rTKA (years) & & $63 \pm 9(24)$ & $63 \pm 8(20)$ & \\
\hline Age at follow-up (years) & & $74 \pm 10(24)$ & $73 \pm 9(20)$ & \\
\hline \multirow[t]{4}{*}{ ASA classification } & ASA 1 & $0 \%$ & $0 \%$ & \\
\hline & ASA 2 & $75 \%(18 / 24)$ & $75 \%(15 / 20)$ & \\
\hline & ASA 3 & $25 \%(6 / 24)$ & $25 \%(5 / 20)$ & \\
\hline & ASA 4 & $0 \%$ & $0 \%$ & \\
\hline BMI $\left(\mathrm{kg} / \mathrm{m}^{2}\right)$ & & $29 \pm 4(24)$ & $30 \pm 5(20)$ & \\
\hline \multirow[t]{2}{*}{ Affected side } & Left & $63 \%(15 / 24)$ & $60 \%(12 / 20)$ & \\
\hline & Right & $37 \%(9 / 24)$ & $40 \%(8 / 20)$ & \\
\hline Time to revision after index TKA (years) & & $6 \pm 7(24)$ & $5 \pm 4(20)$ & \\
\hline \multirow[t]{5}{*}{ Causes of rTKA } & Loosening & $29 \%(7 / 24)$ & $45 \%(9 / 20)$ & \\
\hline & Infection & $4 \%(1 / 24)$ & $55 \%(11 / 20)$ & \\
\hline & Persistent pain & $38 \%(9 / 24)$ & $0 \%$ & \\
\hline & Instability & $17 \%(4 / 24)$ & $0 \%$ & \\
\hline & Component malrotation & $12 \%(3 / 24)$ & $0 \%$ & \\
\hline Re-rTKA & & $13 \%(3 / 24)$ & $15 \%(3 / 20)$ & \\
\hline Re-Re-rTKA & & $33 \%(1 / 3)$ & $33 \%(1 / 3)$ & \\
\hline Mean follow-up (years) & & $11 \pm 3(24)$ & $10 \pm 3(20)$ & \\
\hline \multirow[t]{3}{*}{ Implant at follow-up } & Standard implant & $25 \%(6 / 24)$ & $5 \%(1 / 20)$ & \\
\hline & condylar constrained prosthesis & $54 \%(13 / 24)$ & $60 \%(12 / 20)$ & \\
\hline & Hinged prosthesis & $21 \%(5 / 24)$ & $35 \%(7 / 20)$ & \\
\hline \multirow[t]{3}{*}{ HSS score } & & $81 \pm 10(24)$ & $63 \pm 13(4)$ & \\
\hline & HSS objective & $41 \pm 5(24)$ & $40 \pm 4(4)$ & \\
\hline & HSS symptoms & $40 \pm 8(24)$ & $38 \pm 12(20)$ & 0.748 \\
\hline \multirow[t]{3}{*}{ KSS objective score } & & $80 \pm 13.38(24)$ & $62 \pm 22(4)$ & \\
\hline & KSS objective & $64 \pm 12(24)$ & $51 \pm 24(4)$ & \\
\hline & KSS symptoms & $15 \pm 5(24)$ & $17 \pm 7(20)$ & 0.338 \\
\hline \multirow[t]{3}{*}{ KSS expectation and satisfaction score } & & $34 \pm 9(24)$ & $31 \pm 14(20)$ & 0.346 \\
\hline & KSS satisfaction & $24 \pm 8(24)$ & $23 \pm 10(20)$ & 0.884 \\
\hline & KSS expectation & $10 \pm 3(24)$ & $7 \pm 4(20)$ & 0.062 \\
\hline \multirow[t]{5}{*}{ KSS functional Activity score } & & $52 \pm 19(24)$ & $47 \pm 22(20)$ & 0.524 \\
\hline & KSS walking and standing & $19 \pm 10(24)$ & $18 \pm 10(20)$ & 0.468 \\
\hline & KSS standard activities & $18 \pm 6(24)$ & $17 \pm 8(20)$ & 0.603 \\
\hline & KSS advanced activities & $7 \pm 6(24)$ & $7 \pm 6(20)$ & 0.930 \\
\hline & KSS discretionary activities & $8 \pm 5(24)$ & $6 \pm 4(20)$ & 0.256 \\
\hline OKS $(12=$ best result, $60=$ worst result $)$ & & $28 \pm 6(24)$ & $32 \pm 11(20)$ & 0.137 \\
\hline EQ-5D VAS & & $56 \pm 21(24)$ & $49 \pm 29(20)$ & 0.474 \\
\hline EQ-5D index (Reference: German value set) & & $0.694 \pm 0.112(24)$ & $0.595 \pm 0.218(20)$ & 0.123 \\
\hline
\end{tabular}

$\mathrm{ASA}=$ American society of anesthesiologists; $\mathrm{BMI}=$ Body mass index; TKA = Total knee arthroplasty; rTKA = Revision total knee arthroplasty; $\mathrm{ROM}=$ Range of motion; HSS $=$ Hospital for special surgery score; KSS = Knee society score; OKS = Oxford knee score; $\mathrm{EQ}-5 \mathrm{D}=\mathrm{Measure}$ of health-related quality of life

to range between $83-90$ points following primary TKA [25, 34]. Lee et al. [19] reported an HSS score of 79 after revision TKA due to periprosthetic joint infection and 85 in the aseptic treatment group. Our data indicate a similar outcome with an HSS value of 78 matching this range. The OKS score reported outcome scores following revision TKA range between 23 and 32 points [1, 4, 10, 21]. The here reported population reached a score of 30 , indicating 
Table 3 Demographic data, radiological and functional outcome aseptic vs. septic revisions

\begin{tabular}{|c|c|c|c|c|}
\hline & & Aseptic & Septic & $p$ \\
\hline $\mathrm{N}$ & & 32 & 12 & \\
\hline \multirow[t]{2}{*}{ Gender } & Female & $56 \%(18 / 32)$ & $33 \%(4 / 12)$ & \\
\hline & Male & $44 \%(14 / 32)$ & $67 \%(8 / 12)$ & \\
\hline Age at index surgery (years) & & $57 \pm 8(32)$ & $61 \pm 9(12)$ & \\
\hline Age at rTKA (years) & & $63 \pm 8(32)$ & $64 \pm 8(12)$ & \\
\hline Age at follow-up (years) & & $73 \pm 9(32)$ & $75 \pm 11(12)$ & \\
\hline \multirow[t]{4}{*}{ ASA classification } & ASA 1 & $0 \%$ & $0 \%$ & \\
\hline & ASA 2 & $79 \%(25 / 32)$ & $67 \%(8 / 12)$ & \\
\hline & ASA 3 & $21 \%(7 / 32)$ & $33 \%(4 / 12)$ & \\
\hline & ASA 4 & $0 \%$ & $0 \%$ & \\
\hline $\mathrm{BMI}\left(\mathrm{kg} / \mathrm{m}^{2}\right)$ & & $29 \pm 4(32)$ & $29 \pm 6(12)$ & \\
\hline \multirow[t]{2}{*}{ Affected side } & Left & $59 \%(19 / 32)$ & $67 \%(8 / 12)$ & \\
\hline & Right & $41 \%(13 / 32)$ & $33 \%(4 / 12)$ & \\
\hline Time to revision after index TKA (years) & & $6 \pm 6(32)$ & $3 \pm 2(12)$ & \\
\hline \multirow[t]{5}{*}{ Causes of rTKA } & Loosening & $50 \%(16 / 32)$ & $0 \%$ & \\
\hline & Infection & $0 \%$ & $100 \%(12 / 12)$ & \\
\hline & Persistent pain & $28 \%(9 / 32)$ & $0 \%$ & \\
\hline & Instability & $13 \%(4 / 32)$ & $0 \%$ & \\
\hline & Component malrotation & $9 \%(3 / 32)$ & $0 \%$ & \\
\hline \multirow[t]{2}{*}{ Approach } & One-stage & $72 \%(23 / 32)$ & $8 \%(1 / 12)$ & \\
\hline & Two-stage & $28 \%(9 / 32)$ & $92 \%(11 / 12)$ & \\
\hline Mean follow-up (years) & & $10 \pm 3(32)$ & $11 \pm 4(12)$ & \\
\hline \multirow[t]{3}{*}{ Implant at follow-up } & Standard implant & $19 \%(6 / 32)$ & $8 \%(1 / 12)$ & \\
\hline & Condylar constrained prosthesis & $56 \%(18 / 32)$ & $59 \%(7 / 12)$ & \\
\hline & Hinged prosthesis & $25 \%(8 / 32)$ & $33 \%(4 / 12)$ & \\
\hline \multirow[t]{3}{*}{ HSS score } & & $78 \pm 12(27)$ & $84(1)$ & \\
\hline & HSS objective & $41 \pm 5(27)$ & $44(1)$ & \\
\hline & HSS symptoms & $39 \pm 10(32)$ & $38 \pm 8(12)$ & 0.625 \\
\hline \multirow[t]{3}{*}{ KSS objective score } & & $77 \pm 16(27)$ & $85(1)$ & \\
\hline & KSS objective & $62 \pm 15(27)$ & $71(1)$ & \\
\hline & KSS symptoms & $16 \pm 6(32)$ & $17 \pm 6(12)$ & 0.624 \\
\hline \multirow[t]{3}{*}{ KSS expectation and satisfaction score } & & $34 \pm 10(32)$ & $29 \pm 14(12)$ & 0.241 \\
\hline & KSS satisfaction & $25 \pm 8(32)$ & $22 \pm 11(12)$ & 0.413 \\
\hline & KSS expectation & $9 \pm 3(32)$ & $7 \pm 3(12)$ & 0.108 \\
\hline \multirow[t]{5}{*}{ KSS functional activity score } & & $51 \pm 22(32)$ & $45 \pm 13(12)$ & 0.275 \\
\hline & KSS walking and standing & $19 \pm 10(32)$ & $17 \pm 7(12)$ & 0.381 \\
\hline & KSS standard activities & $18 \pm 7(32)$ & $16 \pm 7(12)$ & 0.437 \\
\hline & KSS advanced activities & $7 \pm 6(32)$ & $5 \pm 4(12)$ & 0.500 \\
\hline & KSS discretionary activities & $7 \pm 5(32)$ & $7 \pm 3(12)$ & 0.850 \\
\hline OKS $(12=$ best result, $60=$ worst result $)$ & & $29 \pm 9(32)$ & $33 \pm 7(12)$ & 0.183 \\
\hline EQ-5D VAS & & $55 \pm 24(32)$ & $48 \pm 29(12)$ & 0.515 \\
\hline EQ-5D index (Reference: German value set) & & $0.661 \pm 0.182(32)$ & $0.618 \pm 0.151(12)$ & 0.322 \\
\hline
\end{tabular}

$\mathrm{ASA}=$ American society of anesthesiologists; $\mathrm{BMI}=$ Body mass index; TKA = Total knee arthroplasty; rTKA = Revision total knee arthroplasty; $\mathrm{ROM}=$ Range of motion; HSS $=$ Hospital for special surgery score; KSS = Knee society score; OKS = Oxford knee score; EQ-5D = Measure of health-related quality of life

comparable patient satisfaction as previously reported. The here reported ROM was $114^{\circ}$ while ranging from 76 to $112^{\circ}$ in the literature [24].
This cohort reached a mean of 0.649 for the EQ-5D index and 53 for the EQ-5D VAS. Concerning the EQ-5D index, Baker et al. reported a score of 0.541 following revision 
TKA in a cohort of 797 patients. Like our cohort, all patients suffered from moderate to severe systemic diseases (ASA II-III, Table 1). While the mentioned group reported a mean follow-up of 7 months, our data represents a followup of 11 years. The extended time frame might explain the slightly superior outcome after revision TKA in our cohort because function and satisfaction relevantly improve during the first 12 months after surgery. Concerning the EQ-5D VAS values, no representative data are available. Therefore, our values are compared to the biggest neighbor-country Germany, where the age group of 65-74 years reaches an EQ-5D VAS score of 69 [15]. Compared to this population, the here reported cohort reached a $22 \%$ lower VAS score. However, looking at the ASA score, our cohort might not be comparable with Germany's average patient health status in that age group. Nevertheless, a lower VAS score is plausible in a cohort of patients with rTKA.

With respect to the subgroup comparison, we did not observe any significant functional differences between a one-stage and a two-stage approach following rTKA. While some authors report similar findings [24], others indicate superior functional outcomes following rTKA by a one-stage approach [23]. Therefore, ongoing randomized clinical trials aim to identify advantages of one-stage vs. two-stage strategies [20]. A two-stage procedure is traditionally favored for management of infected TKA. To analyze whether infection influences the outcome of such two-stage approaches, we compared two-stage rTKA due to infection vs. loosening. In doing so, we did not observe any significant differences between the two subgroups. Similar results have been reported in the past [33], but there is also some evidence showing worse results of infected rTKA compared to not infected rTKA [32]. This study has several limitations. Firstly, it is a small series of included patients, which makes subgroup analysis difficult. Secondly, the here reported population falls in the ASA II and III groups and might not represent patients' average health status requiring a revision TKA. This possible selection bias might even be even intensified by the fact that rather complex cases are admitted to our university center, while standard case are treated in peripheral hospitals. Therefore, outcomes of rTKA in standard cases without significant comorbidities are assumed to be better than reported here. This might be the reason for the discrepancy in the literature while comparing outcomes of primary and revision TKA [30]. Finally, further interesting subgroup analysis regarding stability, ROM, loosening and function of the different implants could not be analyzed due to the limited number in each subgroup. A subgroup of patients was not willing to visit our outpatient department for physical and radiological examination at the final follow-up. Therefore, of this cohort only PROMs and survival could be raised. The reason for this is a long follow-up in an elderly cohort, for whom it is often a burden to attend medical appointments. Data of physical and radiological examination would have added some value, but the study question that focused on PROM and survival could be answered with the raised data. Despite these limitations, we believe that this work adds value to the scientific knowledge about rTKA because of the long follow-up period and the high number of PROMS obtained.

This study can be used to educate patients who are about to undergo the first revision of TKA and to inform them about the possible outcome. Despite a generally good outcome, patients have to be informed about the possibility of revision and/or implant removal, as this is very burdensome for those affected. Furthermore, given a non-significant difference between the outcomes of one- and two-stageexchanges, the indication for a two-stage-exchange must be strictly defined. This is in concordance with a systematic review comparing one- and two-stage-exchange rTKA due to infection [23].

\section{Conclusion}

In our 11 years follow-up, we obtained $89 \%$ implant survivorship. Measurements regarding functional outcome and pain showed results in the medium range of the respective scores, while patient satisfaction lay in the upper third. No significant differences in outcome scores between one- and two-stage revisions and septic versus aseptic revisions were observed.

Authors' contribution A.H. and H-J.A.P. raised the data and wrote the first manuscript draft. F.M.K. designed the study and supervised. S.W. did the statistical analysis and helped to draft the manuscript. The final manuscript was corrected and approved by all authors.

Funding Open access funding provided by University of Bern.

\section{Declarations}

Conflict of interest The authors have no competing interests to declare that are relevant to the content of this article.

Ethical approval Local review board that approved the study: Cantonal ethics-commission Bern Application BASEC-Nr. 2020-01559.

Open Access This article is licensed under a Creative Commons Attribution 4.0 International License, which permits use, sharing, adaptation, distribution and reproduction in any medium or format, as long as you give appropriate credit to the original author(s) and the source, provide a link to the Creative Commons licence, and indicate if changes were made. The images or other third party material in this article are included in the article's Creative Commons licence, unless indicated otherwise in a credit line to the material. If material is not included in the article's Creative Commons licence and your intended use is not 
permitted by statutory regulation or exceeds the permitted use, you will need to obtain permission directly from the copyright holder. To view a copy of this licence, visit http://creativecommons.org/licenses/by/4.0/.

\section{References}

1. Bin Abd Razak HR, Lee JHM, Tan SM, Chong HC, Lo NN, Yeo SJ (2020) Satisfaction rates are low following revision total knee arthroplasty in asians despite improvements in patient-reported outcome measures. J Knee Surg 33:1041-1046. https://doi.org/ 10.1055/s-0039-1692629

2. Bonanzinga T, Tanzi G, Iacono F, Ferrari MC, Marcacci M (2017) Periprosthetic knee infection: two stage revision surgery. Acta Biomed 88:114-119. https://doi.org/10.23750/abm.v88i4S.6802

3. Choi HR, Bedair H (2014) Mortality following revision total knee arthroplasty: a matched cohort study of septic versus aseptic revisions. J Arthroplasty 29:1216-1218. https://doi.org/10. 1016/j.arth.2013.11.026

4. Clement ND, Macdonald D, Burnett R (2013) Predicting patient satisfaction using the Oxford knee score: where do we draw the line? Arch Orthop Trauma Surg 133:689-694. https://doi.org/ 10.1007/s00402-013-1728-3

5. Dawson J, Fitzpatrick R, Murray D, Carr A (1998) Questionnaire on the perceptions of patients about total knee replacement. J Bone Joint Surg Br 80:63-69. https://doi.org/10.1302/ 0301-620x.80b1.7859

6. Delanois RE, Mistry JB, Gwam CU, Mohamed NS, Choksi US, Mont MA (2017) Current epidemiology of revision total knee arthroplasty in the United States. J Arthroplasty 32:2663-2668. https://doi.org/10.1016/j.arth.2017.03.066

7. Devlin NJ, Brooks R (2017) EQ-5D and the EuroQol group: past, present and future. Appl Health Econ Health Policy 15:127-137. https://doi.org/10.1007/s40258-017-0310-5

8. Evans JT, Walker RW, Evans JP, Blom AW, Sayers A, Whitehouse MR (2019) How long does a knee replacement last? A systematic review and meta-analysis of case series and national registry reports with more than 15 years of follow-up. Lancet 393:655-663. https://doi.org/10.1016/S0140-6736(18)32531-5

9. Girerd D, Parratte S, Lunebourg A, Boureau F, Ollivier M, Pasquier G et al (2016) Total knee arthroplasty revision with trabecular tantalum cones: preliminary retrospective study of 51 patients from two centres with a minimal 2-year follow-up. Orthop Traumatol Surg Res 102:429-433. https://doi.org/10. 1016/j.otsr.2016.02.010

10. Hamilton DF, Simpson PM, Patton JT, Howie CR, Burnett R (2017) Aseptic revision knee arthroplasty with total stabilizer prostheses achieves similar functional outcomes to primary total knee arthroplasty at 2 years: a longitudinal cohort study. J Arthroplasty 32:1234-1240.e1231. https://doi.org/10.1016/j. arth.2016.10.028

11. Inacio MCS, Paxton EW, Graves SE, Namba RS, Nemes S (2017) Projected increase in total knee arthroplasty in the United States-an alternative projection model. Osteoarthritis Cartilage 25:1797-1803. https://doi.org/10.1016/j.joca.2017. 07.022

12. Irrgang JJ, Anderson AF (2002) Development and validation of health-related quality of life measures for the knee. Clin Orthop Relat Res (1976-2007) 402:95-109

13. Iwuchukwu C, Wright D, Sofine A, Schwarzkopf R (2016) Can a total knee arthroplasty perioperative surgical home close the gap between primary and revision TKA outcomes? Am J Orthop (Belle Mead NJ) 45:E458-E464

14. Jacobs CA, Christensen CP (2009) Correlations between knee society function scores and functional force measures. Clin Orthop Relat Res 467:2414-2419. https://doi.org/10.1007/ s11999-009-0811-0

15. Janssen MF, Szende A, Cabases J, Ramos-Goni JM, Vilagut G, Konig HH (2019) Population norms for the EQ-5D-3L: a cross-country analysis of population surveys for 20 countries. Eur J Health Econ 20:205-216. https://doi.org/10.1007/ s10198-018-0955-5

16. Kapadia BH, McElroy MJ, Issa K, Johnson AJ, Bozic KJ, Mont MA (2014) The economic impact of periprosthetic infections following total knee arthroplasty at a specialized tertiary-care center. J Arthroplasty 29:929-932. https://doi.org/10.1016/j.arth.2013.09. 017

17. Kumar N, Yadav C, Raj R, Anand S (2014) How to interpret postoperative X-rays after total knee arthroplasty. Orthop Surg 6:179-186. https://doi.org/10.1111/os.12123

18. Kurtz SM, Lau E, Ong K, Zhao K, Kelly M, Bozic KJ (2009) Future young patient demand for primary and revision joint replacement: national projections from 2010 to 2030. Clin Orthop Relat Res 467:2606-2612. https://doi.org/10.1007/ s11999-009-0834-6

19. Lee DH, Lee SH, Song EK, Seon JK, Lim HA, Yang HY (2017) Causes and clinical outcomes of revision total knee arthroplasty. Knee Surg Relat Res 29:104-109. https://doi.org/10.5792/ksrr. 16.035

20. Lindberg-Larsen M, Odgaard A, Fredborg C, Schrøder HM (2021) One-stage versus two-stage revision of the infected knee arthroplasty-a randomized multicenter clinical trial study protocol. BMC Musculoskelet Disord 22:175. https://doi.org/10. 1186/s12891-021-04044-8

21. Minator Sajjadi M, Keyhani S, Kazemi SM, Hanafizadeh B, Ebrahimpour A, Banasiri M (2019) Patient satisfaction following total knee arthroplasty: comparison of short-term results in rheumatoid arthritis and osteoarthritis. Arch Bone Jt Surg 7:61-66

22. Moya-Angeler J, Bas MA, Cooper HJ, Hepinstall MS, Rodriguez JA, Scuderi GR (2017) Revision arthroplasty for the management of stiffness after primary TKA. J Arthroplasty 32:1935-1939. https://doi.org/10.1016/j.arth.2017.01.010

23. Nagra NS, Hamilton TW, Ganatra S, Murray DW, Pandit H (2016) One-stage versus two-stage exchange arthroplasty for infected total knee arthroplasty: a systematic review. Knee Surg Sports Traumatol Arthrosc 24:3106-3114. https://doi.org/10.1007/ s00167-015-3780-8

24. Pangaud C, Ollivier M, Argenson JN (2019) Outcome of singlestage versus two-stage exchange for revision knee arthroplasty for chronic periprosthetic infection. EFORT Open Rev 4:495-502. https://doi.org/10.1302/2058-5241.4.190003

25. Park SJ, Jung YB, Jeong HJ, Shin HK, Jung HJ, Lim JJ et al (2010) Long-term results of primary total knee arthroplasty with and without patellar resurfacing. Acta Med Okayama 64:331-338. https://doi.org/10.18926/amo/40509

26. Postler A, Lützner C, Beyer F, Tille E, Lützner J (2018) Analysis of total knee arthroplasty revision causes. BMC Musculoskelet Disord 19:55. https://doi.org/10.1186/s12891-018-1977-y

27. Robertsson O, Dunbar M, Pehrsson T, Knutson K, Lidgren L (2000) Patient satisfaction after knee arthroplasty: a report on 27,372 knees operated on between 1981 and 1995 in Sweden. Acta Orthop Scand 71:262-267. https://doi.org/10.1080/00016 4700317411852

28. Rosso F, Cottino U, Dettoni F, Bruzzone M, Bonasia DE, Rossi R (2019) Revision total knee arthroplasty (TKA): mid-term 
outcomes and bone loss/quality evaluation and treatment. J Orthop Surg Res 14:280. https://doi.org/10.1186/s13018-019-1328-1

29. Siqueira MB, Klika AK, Higuera CA, Barsoum WK (2015) Modes of failure of total knee arthroplasty: registries and realities. J Knee Surg 28:127-138. https://doi.org/10.1055/s-0034-1396014

30. Stirling P, Middleton SD, Brenkel IJ, Walmsley PJ (2020) Revision total knee arthroplasty versus primary total knee arthroplasty: a matched cohort study. Bone Jt Open 1:29-34. https://doi.org/10. 1302/2633-1462.13.BJO-2019-0001.R1

31. Thiele K, Perka C, Matziolis G, Mayr HO, Sostheim M, Hube R (2015) Current failure mechanisms after knee arthroplasty have changed: polyethylene wear is less common in revision surgery. J Bone Joint Surg Am 97:715-720. https://doi.org/10.2106/jbjs.M. 01534

32. van Kempen RW, Schimmel JJ, van Hellemondt GG, Vandenneucker H, Wymenga AB (2013) Reason for revision TKA predicts clinical outcome: prospective evaluation of 150 consecutive patients with 2-years followup. Clin Orthop Relat Res 471:22962302. https://doi.org/10.1007/s11999-013-2940-8

33. van Rensch PJH, Hannink G, Heesterbeek PJC, Wymenga AB, van Hellemondt GG (2020) Long-term outcome following revision total knee arthroplasty is associated with indication for revision. J Arthroplasty 35:1671-1677. https://doi.org/10.1016/j.arth.2020. 01.053

34. Zhang Z, Chai W, Zhao G, Zhang Q, Chen Z, Wang X et al (2021) Association of HSS score and mechanical alignment after primary TKA of patients suffering from constitutional varus knee that caused by combined deformities: a retrospective study. Sci Rep 11:3130. https://doi.org/10.1038/s41598-021-81285-6

Publisher's Note Springer Nature remains neutral with regard to jurisdictional claims in published maps and institutional affiliations. 